\title{
A REVIEW ON: ATRIGEL-THE MAGICAL TOOL
}

\author{
SALI S. R., GONDKAR S. B., SAUDAGAR R. B.
}

Department of Pharmaceutics, R. G. Sapkal College of Pharmacy, Anjaneri, Nashik 422213, Maharashtra, India Email: shubhamsali44@gmail.com

Received: 25 Dec 2017, Revised and Accepted: 05 Feb 2018

\begin{abstract}
The widely effective and most common form of drug delivery is parenteral administration for active drug substances with poor bio-availability and the drugs with a narrow therapeutic index. Though parenteral administration of drug is often critical and associated with problems such as limited number of acceptable excipients, stringent requirements of aseptic production process, safety issues, patient noncompliance. Still this route maintains its value due to special advantages like quicker onset of action in case of emergency, target the drug quickly to desired site of action, prevention of first pass metabolism etc. The application of advanced drug delivery technology to parenteral administration lead to development of liposomes, nanosuspensions, solid implants etc. to overcome limitations of conventional parenteral delivery. Solid implants are reported to produce very reproducible release profiles. However, because of their size, they require surgical implantation or the use of large trochars to administer the product. Delivery systems consisting of microparticles can be injected into the body using conventional needles and syringes and have been the most widely accepted biodegradable polymer system for parenteral use. However, the manufacturing processes for microparticles are often complex and difficult to control leading to batch-to-batch product non uniformity. These methods of administration often limit the product's market potential due to patient and physician acceptance issues. Therefore, a delivery system that combines the simplicity and reliability of solid implant devices along with convenience and ease of administration of microparticles is desired. In situ gel forming systems represent a desired alternate. This article gives the idea about In situ gel forming system to provide drug release in sustained release manner.
\end{abstract}

Keywords: Parenteral controlled delivery systems, Atrigel, Biodegradable polymers, Implants, Liposomes, in situ gel forming systems

(C) 2018 The Authors. Published by Innovare Academic Sciences Pvt Ltd. This is an open access article under the CC BY license (http://creativecommons.org/licenses/by/4.0/) DOI: http://dx.doi.org/10.22159/ijcpr.2018v10i2.25890

\section{INTRODUCTION}

As number of drug delivery systems has been discovered over the years, parenteral drug delivery system being one of them. Parenteral drug delivery refers to administration by injection which takes the drug directly into the tissue fluid or blood without having to cross the intestinal mucosa. The limitations of oral route are circumvented. Action is faster and surer (valuable in emergency). Gastric irritation and vomiting is not provoked. It can be employed even in unconscious, uncooperative or vomitose patient. There are no chances of interference by food or digestive juices. Liver is also bypassed by this route [1]. But this route specifically requires that the drug delivery system should be sterile, besides being invasive and painful, assistance of other person often being required (though self injection is possible, e. g. insulin by diabetics), there are chances of local injury and being more risky. Once administered, the action is difficult to revert back in case of side effects or toxicity. The different parenteral routes are subcutaneous, Intravenous, Intramuscular, Intra dermal and Intraperitonial.

\section{Parenteral controlled drug delivery systems}

The advanced drug delivery technology can reduces the total number of injection throughout the drug therapy period will be truly advantageous not only in terms of compliance, but also for potential to enhance the quality of the therapy. In such reduction in frequency of drug dosing is achieved, in practice, by the use of specific formulation technologies that guarantee that the release of the active drug substance happens in a slow and predictable manner [2].

In principle, there are three ways to achieve prolonged release of parenteral dosage form. These are-pharmacological, chemical, and physical methods. Pharmacological methods include intramuscular or subcutaneous administration instead of intravenous; the simultaneous administration of vasoconstrictors (adrenalin in local anaesthetics; ephedrine in heparin solutions); and blocking the elimination of drugs through the kidneys by simultaneous administration of a blocking agent, such as probenecid with penicillin or p-amino salicylic acid. Chemical methods include the use of salts, esters, and complexes of the active ingredient with low solubility. Physical methods include the selection of the proper vehicle, thereby giving prolonged release (use of oleaginous solutions instead of aqueous solutions); the addition of macromolecules that increase viscosity (CMC, NaCMC, PVP, tragacanth, etc.); the use of swelling materials to increase viscosity in oleaginous solutions (aluminium monostearate); the additions of adsorbents; the use of solutions from which, upon administration, the drug is precipitated when it contacts body fluids; the use of aqueous and oleaginous suspensions; and the use of implants [3]

\section{Types of parenteral controlled drug delivery systems}

- Surgical implants

- Microspheres

- Liposomes

- Injectable gels

Biodegradable polymers are made from surgical implants by using well-controlled manufacturing processes, such as extrusion, injection moulding, and compression moulding.

These kind of devices usually have very reproducible release profiles. However, because of their size, they require surgical implantation which is often limits the product's market potential due to patient and physician acceptance issues.

Microspheres are developed for parenteral delivery, on the other hand, it can be injected into the body by using conventional needles and syringes. Thus, they have been the most widely accepted biodegradable polymer system for parenteral uses. However, the manufacturing processes for microspheres are often complex and difficult to control. As a result, there are often questions involving costs and batch-to-batch product uniformity [4, 5].

Liposome's on the other hand are versatile carriers for both hydrophilic and lipophilic drug molecules but suffer from several disadvantages such as, high production cost, leakage of drug, short half life and low solubility [6]. 
Biodegradable injectable in situ gel forming drug delivery systems holds the attractive alternative to microspheres and implants as parenteral systems. It consists of biodegradable polymers dissolved in a biocompatible carrier. When the liquid polymer system is placed in the body using standard needles and syringes, it solidifies upon contact with aqueous body fluids to form solid implant. If a drug is injected into the polymer solution, it becomes entrapped within polymer matrix as it gets solidifies. Drug release occurs over time as polymer biodegrades. Biodegradable polymers used in these systems are Polyhydroxyacids, polyanhydrides, polyorthoesters, polyester amides and others. Their importance will grow as numerous proteins will lose their patent protection in the upcoming future [7].

\section{The atrigel drug delivery system}

The Atrigel delivery system can be used for both parenteral and site specific drug delivery. Atrigel system was initially developed by Dunn and co-workers at Southern Research Institute in Birmingham, Alabama in 1987. The technology was licensed to Vipont Research Laboratories (which then became Atrix Laboratories) for the sub gingival delivery of antimicrobials to treat periodontal disease [8]. This system governes many advantages over conventional methods of drug administration including tablets, capsules etc [9].

These include-

\section{-Compatibility with a broad range of pharmaceutical compounds}

Water soluble and insoluble compounds and high and low molecular weight compounds like peptides and proteins, vaccines and natural products can be easily administered by Atrigel systems.

\section{-Less invasive technique}

The application is less invasive and painful compared to implants, which require local anaesthesia and a small surgical intervention.

\section{-Direct delivery to a target area}

Thus helps in achieving higher drug concentrations at the desired site of action to minimize systemic side effects.

\section{-Protection of drug}

Development of an Atrigel drug delivery system of a protein drug helps in preventing denaturation of protein in body fluids.

\section{Sustained drug release}

Helps in reduction of dose, achieve release for extended periods, so there is increase in patient compliance, important for those protein drugs having narrow therapeutic indices.

\section{Biodegradable and biocompatible}

Atrigel system is made of biodegradable polymers and biocompatible solvents so do not require removal.

\section{Economic factors}

Microspheres have to be washed and isolated after preparation; operating expenses for the production of in situ forming applications are marginal, thus lowering investment and manufacturing costs. The technology for the Atrigel system is protected by 33 patents in the United States and 35 patents in the rest of the world. These patents cover the basic technology as well as process improvements [10].

\section{Formulation and development}

The formulation of these systems includes the dissolution of the water insoluble biodegradable polymer into a biocompatible solvent. The drug is next added to the solution where it dissolves or forms a suspension. This drug/Polymer mixture is then easily and conveniently injected into the body where it forms a solid implant inside the tissue. Most frequently used polymers are poly (dllactide), lactide/glycolide copolymers, and lactide/caprolactone copolymers because of their degradation characteristics and their approval by the Food and Drug Administration (FDA). These offer advantage that breakdown products are natural, biocompatible so no problem of toxicity. Various rates of biodegradation can be obtained depending on type of polymer, there combination and ratio [11]. Polymer concentrations ranging from 10 to $80 \%$ by weight are used for preparation of Atrigel drug delivery system [8]. The low molecular weight polymers at low polymer concentrations can be easily injected into the body using standard needles, and they can also be aerosolized for spray applications. The high molecularweight polymers at high polymer concentrations may be used as gels or either putties that can be situated into sites in the body where they solidify and gives support. Some examples are depicted in table 1.

Table 1: Biodegradation time of different biodegradable polymers [11]

\begin{tabular}{ll}
\hline Polymer & Time of biodegradation \\
\hline Poly Lactide & $28-24$ Months \\
Poly dl Lactide & $12-16$ Months \\
50:50 Lactide/Glycolide & $50-60$ Months \\
85:15Lactide/Glycolide & 5 Months \\
\hline
\end{tabular}

The solvents are introduced in the Atrigel system to get dissolve the polymers of various range from the more hydrophilic solvents such as dimethyl sulfoxide, $\mathrm{N}$-methyl-2-pyrrolidone (NMP), tetraglycol, and glycol furol to the more hydrophobic solvents such as propylene carbonate, triacetin, ethyl acetate, and benzyl benzoate. The most frequently used solvent is NMP because of its solvating ability and its safety/toxicology profile. A Drug Master File (DMF) on this solvent has been filed with the FDA [12].

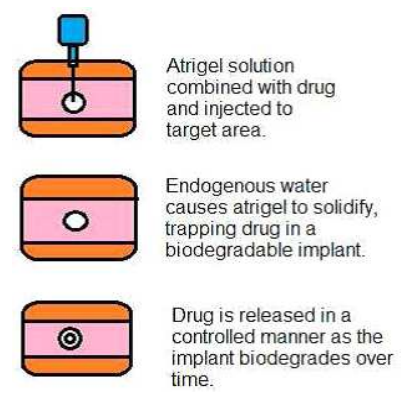

Fig. 1: Controlled release by Atrigel system
Both in vitro and in vivo release studies were used to optimize the release characteristics of the formulations. For the in vitro studies, the drug is combined with the polymer solution and small drops of the mixture (about $50 \mathrm{mg}$ ) are added to phosphate-buffered saline solution. The receiving fluid is replaced at selected times with fresh solution, and the removed phosphate buffer saline solution is analyzed for drug concentration using a variety of analytical methods [13].

\section{Method of manufacturing}

In-situ forming drug delivery systems (ISFD) Injectable in-situ forming implants are classified in to four categories based on the mechanism of achieving solidification in vivo [3].
1. Thermoplastic Paste
2. In-situ cross linking system
3. In-situ polymer precipitation
4. Thermally-induced gelling system
5. In-situ solidifying organogels

\section{Thermoplastic pastes (TP)}

Thermoplastic pastes gives semisolid polymers, in which they are injected to get melt and form a depot upon cooling to body 
temperature. They are characterized as having a low melting point or $\mathrm{Tg}$ (glass transition temperature) in the range of $25-65^{\circ} \mathrm{C}$ and an intrinsic viscosity in the range of $0.05-0.8 \mathrm{dl} / \mathrm{g}$. Below the viscosity of $0.05 \mathrm{dl} / \mathrm{g}$, no delayed release could be observed, where as above $0.8 \mathrm{dl} / \mathrm{g}$ the ISFD was no longer injectable using a needle. At injection temperature above $37^{\circ} \mathrm{C}$ but below $65^{\circ} \mathrm{C}$ these polymers behave like viscous fluids which solidify to highly viscous depots.

\section{In-situ cross-linked polymer systems}

The control on the diffusion of hydrophilic macromolecules is get advantageous by the formation of cross-linked polymer. Also the Cross-linked polymer network can be found in-situ by free radical reactions initiated by heat (thermosets) or absorption of photon or ionic interactions between small cation and polymer anions. Ionmediated gelation has been reported for a number of polymers, e. g. alginates/calcium ions or chitosan/phosphate ions. The concentrations of the counter ion available under physiological conditions are usually insufficient for cross-linking of the above mentioned polymers. Only the calcium concentration in the eye led to in-situ formation of alginate formulations. Despite these applications, there are two important factors which limit the use of calcium alginate. The first factor is their potential immunogenicity and the second is longer time in vivo degradability.

\section{In-situ polymer precipitation}

A water-insoluble and biodegradable polymer is dissolved in a biocompatible organic solvent to which a drug is added forming a solution or suspension after mixing. When this formulation is injected into the body, the water miscible organic solvent dissipates and water penetrates into the organic phase. This tends to phase separation and precipitation of the polymer forming the depot at the site of injection. This method has been designed as Atrigel TM technology, which used as a drug carrier for Eligard TM contains the leuteinizing hormone releasing hormone (LHRH) agonist leuprolide acetate $(7.5,22.5$ or $30 \mathrm{mg}$ ) and poly (lactide-coglycolic acid)(PLGA) $75 / 25$ dissolved in N-methyl-2-pyrrolidone (NMP) in a 45:55 (m/m) polymer: MP ratio. This system led to suppression of testosterone levels in dogs for approximately $91 \mathrm{~d}$. One of the problems with these systems is the possibility of a burst in drug release especially during the first few hours after injection into the body. In order to control the burst effect, four factors have been examined, the concentration of polymer in the solvent, the molecular weight of the polymer, the solvent used and the addition of surfactant. Also the drug burst is directly related to the dynamics of the phase inversion. Brodbeck et al. demonstrated that protein release kinetic from ISFD was influenced by solution thermodynamics, e. g. solvent strength and water miscibility. They studied NMP, triacetin and ethyl benzoate ternary phase systems with PLGA and water. NMP shows rapid phase inversion associated with a high drug burst where as triacetin and ethylbenzoate yielded low phase inversion rates, resulting in a slow gelation which reduced the drug burst of protein significantly. Himmelstein and joshi studied that polymer complex of PEG, polymethacrylic acid (PMA), and polyacrylic acid (PAA) is stable below pHK5.7, the complex is insoluble in water but dissolves in a hydroalcoholic solvent to yield a clear viscous solution. After injection the diffusion of ethanol from the liquid transforms the system into a gel upon contact with physiological condition.

The gel disappears from the site with time due to complex dissociation into water soluble and low molecular weight component, which can be eliminated by glomerular filtration. Carbopol is a pH dependent polymer, which forms a low viscosity gel in alkaline environment (e. g. pH-7.4) and stays in solution in acidic $\mathrm{pH}$. The addition of HPMC, a viscosity inducing agent, to carbopol reduces the carbopol concentration and hence the solution acidity while preserving the viscosity of the in-situ gelling system. This system gels upon an increase in $\mathrm{pH}$ when injected [4].

\section{Thermally induced gelling system}

Many polymers undergo abrupt changes in solubility as a function of environmental temperature. The thermo sensitive polymer, poly ( $\mathrm{N}$ isopropyl acrylamide) [poly (NIPAAM)] exhibit sharp lower critical solution temperature, LCST at about $32{ }^{\circ} \mathrm{C}$. which can be shifted to body temperature by formulating poly NIPAAM based gels with salt and surfactant. Unfortunately, poly NIPAAM is not suitable for biomedical applications due to its wellknown cytotoxicity (activation of platelets) and non-biodegradability. Triblock poly (ethylene oxide)poly (propylene oxide)-poly(ethylene oxide) copolymer, PEO-PPOPEO (pluronics or poloxamers), have shown gelation at body temperature when highly concentrated polymer solution $>15 \% \mathrm{w} / \mathrm{w}$ were injected. These polymer concentration shown disadvantage of changing the osmolarity of the formulation, kinetics of the gelation, and causes discomfort in ophthalmic applications due to vision blurring and crusting. Macro med produced thermo sensitive biodegradable polymers based on $\mathrm{ABA}$ and $\mathrm{BAB}$ triblock copolymers. Where $A$ is hydrophobic polyester block and $B$ denotes the hydrophilic PEG block. The aqueous polymer solution of PEG-PLA-PEG is filled with drug at $45{ }^{\circ} \mathrm{C}$ after injected into animal it form a gel at body temperature, which continuously releasing hydrophilic model substances fluorescein isothiocyanate dextran (FITC-dextran), over 10-20days. Veyries et al. demonstrated the possibility of controlled release of vancomycin from Pluronic F127. They investigated Poloxamer 407 (Pluronic F127) 25\% formulations aimed at prolonging the residence time of vancomycin, a time dependent antibiotic, in a body site with a high infectious risk. It appeared that neither the rheological properties of the Poloxamer matrices nor the antibacterial activity of vancomycin was altered by their combination. Two formulations were prepared, one saturated and one unsaturated (solubilized) with vancomycin. In vitro, the dispersed form (saturated) exhibited prolonged release, with a lower diffusion coefficient of vancomycin compared to the solubilized form

(4.7 X $10-8$ vs. $2.1 \times 10-7 \mathrm{~cm} 2 \mathrm{~s}-1$ ). In rats, a single dose was well tolerated and resulted in a high local concentration for $24 \mathrm{~h}(>131$ $\mathrm{mg} / \mathrm{l}$ ), followed by lower but effective antibacterial levels for at least $8 \mathrm{~d}$. Based on the release profiles, good preservation of vancomycin activity, good tolerability in rats, and ease of administration, it was concluded that Poloxamer 407 might be useful as a vancomycin delivery vehicle for local prophylaxis of infections, especially in prosthetic surgery.

\section{In-situ solidifying organogel}

Organogels are composed of water insoluble amphiphilic lipids, which swell in water and form various types of lyotropic liquid crystals. The amphiphilic lipids examined for drug delivery are glycerol monooleate, glycerol monopalmitostearate, glycerol monolinoleate, sorbitan monostearate (SMS) and different gelation modifiers (polysorbates 20 and 80 ) in various organic solvents and oils. These compound forms a cubic liquid crystal phase upon injection into an aqueous medium which is gel like and highly viscous. SMS organogels containing either $\mathrm{w} / \mathrm{o}$ or vesicular in water in oil (v/w/o) emulsion were investigated in vivo as delivery vesicles for vaccines using albumin (BSA) and haemagglutin (HA) as model antigens. Intramuscular administration of the v/w/o gel yielded the long lasting depot effect (48hr). Gao et al. achieved controlled releases of contraceptive steroids levonorgestrel and ethinyl estradiol. In these work biodegradable organogels formulations prepared from glycerol palmitostearate (precirol) in derivatized vegetable oil, show in vitro release of levonorgestrel up to $14 \mathrm{~d}$.

\section{Atrigels as drug carrier devices}

The most advanced product using Atrigel as a carrier, Eligard, incorporating LHRH agonist

Leuprolide acetate $(7.5,22.5$ or $30 \mathrm{mg})$ and PLGA $75 / 25$ dissolved in $\mathrm{N}$-methyl-2-pyrrolidone (NMP) in a $45: 55(\mathrm{~m} / \mathrm{m})$ polymer: NMP ratio. The carrier system showed reduction in testosterone levels in dogs for approximately $91 \mathrm{~d}$ [14-15].

The in vitro release of doxycycline hyclate from implants formed from three different polymers dissolved in NMP has been studied. The more hydrophobic poly (dl-lactide co-caprolactone) (PLC) showed the slowest release of the drug. The hydrophilic poly (dllactide-co-glycolide) (PLG) lead to low initial release of drug followed by a more rapid release once the polymer becomes hydrated. The poly (dl-lactide) (PLA) showed the highest initial burst of drug followed by a sustained release out to $8 \mathrm{~d}(8)$. 
Polymer molecular weight is reported and its affect the release of drug e. g. in vitro release of naltrexone base from an implant of an Atrigel formulation containing a 50/50 PLG copolymer in NMP was studied. The higher molecular-weight polymer (IV $=0.73 \mathrm{dL} / \mathrm{g}$ ) showed highest burst release of drug whereas the more moderatemolecular-weight polymer (IV $=0.35 \mathrm{dL} / \mathrm{g}$ ) gave and almost zeroorder release of drug (16).

It has been reported that protein release kinetics was also influenced by solution thermodynamics, e. g. solvent strength and water miscibility. The study includes NMP, triacetin and ethyl benzoate as ternary phase systems with PLGA and water. NMP exhibited a rapid phase inversion associated with a high drug burst, due to the formation of a porous rubbery gel structure. In contrast, other solvents, such as triacetin and ethyl benzoate, both weak solvents for PLGA, yielded low phase inversion rates, resulting in a slow gelation which reduced the drug burst of proteins significantly. Therefore, solvent type and polymer concentration are the most critical factors determining the drug release under in vitro and possibly also in vivo conditions $(17,18)$.

0/0 emulsion systems have been fabricated using an internal polymer phase (drug,

biodegradable polymer and organic solvent) and peanut oil as external phase claiming the in situ formation of microspheres at the injection site as an approach to reduce the unwanted local irritation potential of in situ precipitating systems (19).

A comparative study using different hydrophilic and hydrophobic solvent systems for injectable gels was recently reported by Cleland. In this study, homogenous solutions of poly (D, Llactide), PLA, with the protein were obtained when benzyl alcohol/benzyl benzoate mixtures were used (20).

\section{Evaluation and characterization of in-situ gel system}

\section{Clarity}

The clarity of formulated solution determined by visual inspection under black and white background.

\section{Texture analysis}

The firmness, consistency and cohesiveness of formulation are assessed using texture analyzer, which mainly indicates the syringe ability of sol so the formulation can be easily administered in vivo. Higher values of adhesiveness of gels are needed to maintain an intimate contact with surface like tissues.

\section{Sol-Gel transition temperature and gel time}

For in-situ gel forming system incorporating thermo reversible polymer, the sol-gel transition temperature may be defined as that temperature at which the phase transition of sol meniscus is first noted when kept in a sample tube at a specific temperature and then heated at specific rate. Gel formation is indicated by a lack of movement of meniscus on tilting the tube.

\section{Gel strength}

This parameter can be evaluated using a rheometer. It depends on the mechanism of the gelling of gelling agent used; a specific amount of gel is prepared in a beaker, from the sol form. This gel-containing beaker is raised at a certain rate, so pushing a probe slowly through the gel. The change in the load on the probe can be measured as a function of depth on immersion of the probe below gel surface.

\section{Viscosity and rheology}

This is an important parameter for in-situ gels to be evaluated. The viscosity and rheological properties of the polymeric formulation, either in solution or in gel made with artificial tissue fluid were determined with Brookfield rheometer or some other type of viscometer such as Ostwald's viscometer.

\section{Fourier transforms infrared spectroscopy and thermal analysis}

During gelation process, the nature of interacting forces can be evaluated using this technique by employing potassium bromide
(KBr) pellet method. Thermo-gravimetric analysis (TGA) can be conducted for in-situ forming polymeric system to quantitate the percentage of water in hydro gel. Differential Scanning Calorimetry (DSC) is used to observe any change in thermograms as compared with pure ingredients used thus indicates the interaction.

\section{Sterilization and packaging}

Atrigel system is a viscous polymer solution so poses a difficulty in pouring in vials and aspirate into syringes at the time of use.

Therefore, the products currently marketed using this technology are filled into plastic syringes and packaged with foil-lined material to protect from moisture. Atrix Laboratories has developed custommade equipment to fill a variety of plastic syringes with the polymer solutions within narrow fill volumes.

As the drug and polymer are in solution, degradation of both components and reactions between the two may occur somewhat faster with some formulations than in a dry, solid state. With these products, the drug and polymer solution are maintained in separate syringes until use. At the time of use the two syringes are coupled together and the contents are mixed thoroughly by moving the materials back and forth between the two syringes. The homogeneous solution or mixture is drawn into one syringe, the two syringes are decoupled, and a needle is attached for injection. This type of product provides for the maximum stability of the drug as well as the polymer. It also allows the drug to be sterilized by gamma irradiation in a dry state where it is often more stable.

Specific syringe configurations have been developed that enable the two syringes to be connected directly together using luer lock fittings, ensure that when the needle is attached to the syringe with the product, it remains in place during the injection.

Loading of drug into plastic syringes can be done by different ways. One of these techniques is powder filling, where precise control of fill weight is necessary. The equipment for powder filing has been custom designed and fabricated. Second is when the quantity of drug is too small to precisely fill the syringes or if the flow characteristics are not satisfactory, then the drug can be dissolved in water, sterile-filtered, and filled into plastic syringes where the drug can be lyophilized to a dry powder. Filling the polymer into the syringes first involves simply loading the solvent and polymer into a sterile plastic container and placing it on a roll mixer. The polymer solution is then transferred from the plastic container to the syringe-filling equipment where it is loaded into individual syringes. The plastic container can then be discarded and the need for thorough cleaning is eliminated.

The filled syringes are capped and placed into foil-lined packages to prevent moisture absorption. The drug is either powder-filled or lyophilized into syringes. If the drug is stable to gamma irradiation, then terminal sterilization is done by this method. If the drug is not stable to gamma irradiation, then the lyophilization is carried out under aseptic conditions, and the polymer solution is sterilized by gamma irradiation. With this technique, the production of several hundred syringes to thousands in one batch can easily be done. Atrigel system can be sterilized by filtration technique but this method is usually not preferred because of viscosity of this system. Gamma irradiation was evaluated and found to be a convenient method of terminal sterilization of the polymer solution. There is some loss in polymer molecular weight during gamma irradiation, but this is compensated for by using a polymer with a slightly higher molecular weight initially [21].

\section{CONCLUSION}

The injectable in-situ gelling system for prolonged release through parentral delivery ensures that promising system which can control the region where it is required. This explains the method of manufacture, physical charactarisation and etc in detail. These modification, if implemented successfully to the atrigel technology, then it will surely increases its uniqueness and its applicability to the variety of drug delivery products.

\section{AUTHORS CONTRIBUTIONS}

All the author have contributed equally 


\section{CONFLICT OF INTERESTS}

Decleared no

\section{REFERENCES}

1. KD Tripathi. Essentials of medical pharmacology, Medical Publishers, India; 2006.

2. Raghanaveen. Parenteral Controlled Drug Delivery System, Pharmainfo. Net; 2009.

3. JR Robinson, VL Lee. Controlled drug delivery: fundamental and applications, marcel dekker, USA; 2003.

4. M Radomsky, L Liu, Z Iwamoto. Sustained release injectable products, interpharm press: Englewood, Colorado; 2000. p. 181-202.

5. L Lachman, HA Lieberman. The theory and practice of industrial pharmacy, CBS publishers, India; 2005.

6. G Gregoriadis. Liposome technology. Vol. 1. Informa health care, USA; 2007.

7. CB Packhaeuser, J Schnieders, CG Oster, T Kissel. In situ forming parenteral drug delivery systems: an overview. Eur J Pharm Biopharm 2004;58:445-55.

8. R.L. Dunn, J.P. English, D.R. Cowsar, D. P. Vanderbilt. US patent 4, 938,763; 1990.
9. D. B. Patel, Journal of Global Pharma Technology, 2010, vol2, 85-90.

10. N. K. Jain, Controlled and Novel Drug Delivery, CBS Publishers, 2008.

11. A. J. Spiegel, Noseworthy, J.Pharm. Sci. 1963, 52, 917-927.

12. M. Rathbone, J. Hadgraft, M. Roberts, Modified Release Drug Delivery Technology,Marcel Dekker, 2003.

13. F. M. Chu, M. Jayson, M. K. Dineen, R. Perez, R. Harkaway, R. C Tyler, J. Urol., 2002, 168 , 1199-1203.

14. R. L. Dunn, J. S. Garrett, H. Ravivarapu, B. L. Chandrashekar, US Patent 6,565,874,2003.

15. A. J. Tipton, W. U. V. Trumpore, R. L. Dunn, US-Japan Symposium on Drug Delivery, 1991, 16-20.

16. K. J. Brodbeck, J. R. Desnoyer, A. J. Mchugh, J. Control. Release, 1999, $62,333-344$.

17. P. D. Graham, K. J. Brodbeck, A. J. Mchugh, J. Control. Release, 1999, 58, 233-245.

18. H. Kranz, G. A. Brazeau, J. Napaporn, R. L. Martin, W. Millard, R. Bodmeier, Int. J. Pharm., 2001, 212, 11-18.

19. J. L. Cleland, Injectable Gels for Local and Systemic Delivery of Proteins, Proceedings 28th International Symposium on Controlled Release of Bioactive Materials and Fourth Consumer and Diversified Products Conference, Vol. 1, 2001.

20. B. K. Lowe, R. L. Norton, E. L. Keeler, K. R. Frank, A. J. Tipton, Trans Soc Biomater., 1993, 16-54. 